\title{
Predicted MOND velocity dispersions for a catalog of ultra-diffuse galaxies in group environments
}

\author{
Oliver Müller ${ }^{1}$, Benoit Famaey ${ }^{1}$, and Hongsheng Zhao ${ }^{2}$ \\ ${ }^{1}$ Université de Strasbourg, Observatoire Astronomique de Strasbourg (ObAS), CNRS UMR 7550, Strasbourg, France \\ e-mail: oliver.muller@astro.unistra.fr \\ 2 Scottish Universities' Physics Alliance, University of St Andrews, St Andrews, UK \\ Received 18 December 2018 / Accepted 9 January 2019
}

\begin{abstract}
The possibility that ultra-diffuse galaxies are lacking dark matter has recently stimulated interest to check the validity of modified Newton dynamics (MOND) predictions on the scale of such galaxies. It has been shown that the external field effect (EFE) induced by the close-by galaxy can suppress the velocity dispersion of these systems, so that they appear almost dark matter free in the Newtonian context. Here, following up on this, we are making a priori predictions for the velocity dispersion of 22 ultra-diffuse galaxies in the nearby Universe. This sample can be used to test MOND and the EFE with future follow-up measurements. We have constructed a catalog of nearby ultra-diffuse galaxies in galaxy group environments, and set upper and lower limits for the possible velocity dispersion allowed in MOND, taking into account possible variations in the mass-to-light ratio of the dwarf and in the distance to the galaxy group. The prediction for the velocity dispersion is made as a function of the three dimensional separation of the dwarf to its host. In 17 out of 22 cases, the EFE plays a crucial role in the prediction.
\end{abstract}

Key words. galaxies: kinematics and dynamics - galaxies: dwarf - cosmology: theory

\section{Introduction}

In recent years, the number of newly discovered ultra-diffuse galaxies (UDGs) in the nearby Universe has exploded. While it has long been known that this special type of galaxy exists in galaxy cluster environments (e.g., Sandage \& Binggeli 1984; Impey et al. 1988), the discovery of UDGs in nearby galaxy groups (e.g., Crnojević et al. 2014; Merritt et al. 2016; Cohen et al. 2018) has brought new insights, as well as opportunities to better study these systems. Today, UDGs have been defined by their low surface brightness of $>25 \mathrm{mag} \mathrm{arcsec}^{-2}$ in the $V$-band, and large radial extent, with projected half-light radii $r_{\text {eff }}>1.5 \mathrm{kpc}$ (van Dokkum et al. 2015). In morphology, they are not distinguishable from normal dwarf galaxies and appear both as early and late type galaxies (Sandage \& Binggeli 1984). Their formation is still under intense debate with different proposed formation mechanisms. For example it has been argued that these galaxies are failed Milky Way type galaxies (van Dokkum et al. 2015; Yozin \& Bekki 2015), just normal dwarf galaxies affected by stellar feedback, quenching, and outflows (Amorisco \& Loeb 2016; Di Cintio et al. 2017; Chan et al. 2018), or rather a mixed bag of objects (Toloba et al. 2018).

Recently, the UDG NGC 1052-DF2, a putative member of the NGC 1052 galaxy group (Cohen et al. 2018), located at $20 \mathrm{Mpc}$, has caught some attention, following the announcement by van Dokkum et al. (2018) that this galaxy apparently lacks dark matter. This result was inferred from the velocity dispersion of ten bright globular clusters associated with this galaxy. Using a biweight estimator, van Dokkum et al. (2018) derives a velocity dispersion of only $\sigma_{\text {vel }}=3.2 \mathrm{~km} \mathrm{~s}^{-1}$ with a $90 \%$ upper limit of $\sigma_{\text {vel }}=10.5 \mathrm{~km} \mathrm{~s}^{-1}$, which is consistent with the baryonic matter of this galaxy alone. Following a more conservative approach, Martin et al. (2018) obtained a value of $\sigma_{\mathrm{vel}}=9.2 \mathrm{~km} \mathrm{~s}^{-1}$, with a $90 \%$ upper limit of $\sigma_{\mathrm{vel}}=17.2 \mathrm{~km} \mathrm{~s}^{-1}$. While the globular cluster population can be used as a tracer for the mass of the system (Toloba et al. 2018), ultimately, the velocity dispersion of the stellar body should be used. For example, in the Fornax dwarf spheroidal, where both estimates are available, there is a strong discrepancy between the two values (Laporte et al. 2019). We note that, for NGC 1052-DF2 a stellar velocity dispersion has recently been measured using IFU observations with VLT+MUSE (Emsellem et al. 2018; Fensch et al. 2018).

The apparent lack of dark matter in a dwarf galaxy could have some challenging consequences for alternative gravity scenarios such as modified Newton dynamics (MOND; Milgrom 1983), as previously investigated by, for example, Gentile et al. (2007, 2012), Lelli et al. (2015). The argument is that, in MOND, the baryonic matter alone gives the high observed velocities in galaxies, and notably the flat rotation curves in spirals. This argument was indeed brought up by van Dokkum et al. (2018) in the case of NGC 1052-DF2. This simple assessment, however, is only true if the galaxy resides in relative isolation, that is, if no other galaxy is nearby. In the case that there is another gravitational potential close by, its environmental effect has to be taken into account when estimating the internal velocity dispersion, a phenomenon exclusively appearing in MOND. This so-called external field effect (EFE) arises from the non-linearity of the MONDian Poisson equation (Bekenstein \& Milgrom 1984).

The EFE can ultimately lower the velocity dispersion of a system, or in other words, make it Newtonian. Because this EFE appears only in MOND, and not within the standard model of cosmology, it is a highly intriguing feature to test and distinguish these paradigms. For a thorough discussion of the EFE and its consequences, see for example Haghi et al. (2016), Thomas et al. (2018); the supplementary 
materials of Kroupa et al. (2018); or the extensive review of MOND (Famaey \& McGaugh 2012).

For the Andromeda dwarf galaxies in the Local Group, it has been realized that this EFE plays a crucial role, allowing MOND to successfully predict the velocity dispersion of those dwarfs (McGaugh \& Milgrom 2013a,b). For the "feeble-giant" CraterII (Torrealba et al. 2016), an ultra-faint dwarf galaxy satellite of the Milky Way, a very low velocity dispersion has been predicted due to the EFE (McGaugh 2016) and subsequently measured (Caldwell et al. 2017). Several more predictions of velocity dispersions for the ultra-faint dwarf galaxies around the Milky Way (Pawlowski \& McGaugh 2014; Pawlowski et al. 2015; Cortés \& Hernandez 2017) now await follow-up measurements. Taking the EFE into account for NGC 1052-DF2, the expected velocity dispersion was estimated to be $\sigma_{\text {vel }} \simeq$ $14 \mathrm{~km} \mathrm{~s}^{-1}$, consistently estimated by two independent teams (Famaey et al. 2018; Kroupa et al. 2018). While the former used an analytic expression to get this results, the latter invoked an $\mathrm{N}$ body integrator (Londrillo \& Nipoti 2009) to get this estimate, thereby validating the analytic estimate. This result alleviates the claim that NGC 1052-DF2 could falsify MOND, but shows that UDGs are an excellent testbed to study the impact of the EFE. Following up on this previous work, we hereby make a priori predictions for the velocity dispersion of two dozen UDGs in the nearby Universe, thus making MOND and its EFE falsifiable in a currently less-explored regime than that of spiral galaxies. Interestingly, Milgrom $(2018,2019)$ has recently extended the succesful predictions of MOND to the regime of the internal dynamics of galaxy groups themselves.

The paper is organized as follows: in Sect. 2 we discuss how the velocity dispersion can be predicted in MOND, in Sect. 3 we present a catalog of nearby UDGs in galaxy group environments, in Sect. 4 we predict the velocity dispersion for the UDG catalog, and in Sect. 5 we present a brief discussion and estimation on how these values can be measured. Finally, we give our summary and conclusions in Sect. 6.

\section{The external field effect calculation}

The Poisson equation of MOND reads, with Famaey \& Binney (2005) interpolating function:

$\nabla \cdot\left[\mathbf{g} \mu\left(\frac{g}{a_{0}}\right)\right]=4 \pi G \rho, \quad \mu(x)=\frac{x}{1+x}$,

where $\mathbf{g}$ is the gravitational acceleration vector, $\rho$ the baryonic density, and $a_{0}=1.2 \times 10^{-13} \mathrm{~km} \mathrm{~s}^{-2}$ (Begeman et al. 1991) is the MOND acceleration constant marking the transition from the Newtonian to the deep-MOND regime. The fact that $\mu<1$ when in the regime of low acceleration $\left(g<a_{0}\right)$, in which NGC 1052DF2 clearly is, would suggest that such galaxies should always display a "phantom" dark matter behaviour, that is, a dark matter behavior when interpreted in the context of Newtonian dynamics.

However, when the galaxy is not isolated, one should take into account the EFE, such that

$\mathbf{g}=\mathbf{g}_{\mathrm{ex}}-\nabla \phi$

where $\mathbf{g}_{\mathrm{ex}}$ is the gravitational field from the neighbouring galaxies, and $\phi$ the internal MOND potential.

In a MONDian universe, the velocity dispersion of an isolated spherical and isotropic system in the low acceleration regime is easily calculated from its baryonic mass $M$ alone (e.g.,
McGaugh \& Milgrom 2013a)

$\sigma_{\text {iso }}=\left(\frac{4}{81} G M a_{0}\right)^{1 / 4}$,

where $\mathrm{M}$ is the total baryonic mass. This formula applies when the total acceleration (internal plus external acceleration) is well bellow $a_{0}$, i.e., $g+g_{\text {ex }}<a_{0}$.

When the internal acceleration is larger than the external, $g_{\text {ex }}<g<a_{0}$, then we can apply the given formula for the isolated case. However, if $g<g_{\text {ex }}<a_{0}$ the object is quasi-Newtonian, where Newton's law of Gravity applies with a renormalization of the gravitational constant $G$ (McGaugh \& Milgrom 2013a; Famaey et al. 2018). The external acceleration can be approximated by $g_{\mathrm{e}}=v_{\text {rot }}^{2} / D$, where $v_{\text {rot }}$ is the rotation curve of the external galaxy and $D$ is the separation between the two objects. Using Eq. (59) of Famaey \& McGaugh (2012), one can estimate the MOND acceleration $g$ at the halflight radius using

$\left(g+g_{\mathrm{ex}}\right) \mu\left(\frac{g+g_{\mathrm{ex}}}{a_{o}}\right)=g_{\mathrm{N}, 1 / 2}+g_{\mathrm{ex}} \mu\left(\frac{g_{\mathrm{ex}}}{a_{0}}\right)$,

with

$g_{\mathrm{N}, 1 / 2}=\frac{G M}{2 r_{1 / 2}^{2}}$,

where $\mu(x)=x /(1+x)$ is the simple interpolation function (Famaey \& Binney 2005), and $g_{\mathrm{N}, 1 / 2}$ is the Newtonian internal field for the mass embedded within the $3 \mathrm{D}$ deprojected half light radius $\left(r_{1 / 2}=4 / 3 r_{\text {eff }}\right)$. The expression solved for $g$ is rather long, so we refrain from showing it here and refer to the footnote 34 of Famaey \& McGaugh (2012), where it is explicitly given. We can now estimate the true velocity dispersion of the system, corrected for the external field. Having estimated $g$, we can calculate the renormalization of the gravitational constant with

$G_{\text {renorm }}=G \cdot\left(g / g_{\mathrm{N}, 1 / 2}\right)$.

Finally, we can use the mass estimator in Wolf et al. (2010) Eq. (2) to calculate the velocity dispersion:

$\sigma=\sqrt{\frac{0.5 M G_{\text {renorm }}}{3 r_{1 / 2}}}$,

where $0.5 M$ corresponds to the mass embedded within $r_{1 / 2}$. This cooking recipe to calculate the velocity dispersion in the quasiNewtonian regime is discussed in more detail in Famaey et al. (2018), who used it to successfully derive the MONDian value for NGC 1052-DF2 to be between 8.9 and $19.0 \mathrm{~km} \mathrm{~s}^{-1}$ depending on the interpolating function, stellar mass-to-light ratio, and three-dimensional distance to the host. We note that the latest measured velocity dispersion value, using deep MUSE IFU observations (Emsellem et al. 2018) for the stellar body of NGC 1052-DF2 is $\sigma_{\text {stellar }}=16.3 \mathrm{~km} \mathrm{~s}^{-1}$, and for the globular cluster and planetary nebulae population associated to this galaxy is $\sigma_{\mathrm{GC}, \mathrm{PN}}=10.5 \mathrm{~km} \mathrm{~s}^{-1}-$ which are well within the allowed MONDian range for this system.

\section{A catalog of nearby ultra-diffuse galaxies}

\subsection{Data}

Several independent teams have taken up the effort to search for nearby low-surface brightness galaxies in group (e.g., 
Merritt et al. 2014; Carlin et al. 2016; Javanmardi et al. 2016; Bennet et al. 2017; Müller et al. 2015, 2017a; Mihos et al. 2018) and cluster (e.g., Mihos et al. 2015, 2017; van der Burg et al. 2016; Venhola et al. 2017; Eigenthaler et al. 2018) environments. While most detections in these surveys correspond to the regime of the normal dwarf galaxies, several UDGs have also been discovered, based on their integrated light profiles. Up to now, no compilation of these objects exists. Therefore we have collected UDGs from the literature - mainly selecting objects with half-light radius $r_{\text {eff }}$ estimates larger than $1.5 \mathrm{kpc}$ (including those objects with very close values to $1.5 \mathrm{kpc}$, for example, NGC 7814-DGSAT-7 with $r_{\text {eff }}=1.49 \mathrm{kpc}$ ) and residing in a galaxy group environment based on their projected position in the sky. Therefore we excluded UDGs residing in clusters, because in these environments too many things can affect the velocity dispersion of the UDGs. Also, we only consider galaxies in the nearby Universe $(z<0.01)$. While going further would indeed increase the sample (Greco et al. 2018), the galaxy's association to a certain galaxy group becomes more difficult to estimate.

In Table 1, we present our sample of nearby UDGs within galaxy group environments. We have compiled the relevant data needed to estimate the velocity dispersion in MOND (see Sect. 2). For this, we need the total luminosity of the galaxy $L_{\odot}$, the half-light radius $r_{\text {eff }}$, and the projected separation to the host galaxy $\Delta_{\text {proj }}$. When these data were missing, we derived values from the published data, for example, the mean surface brightness from the apparent magnitude and the half-light radius, the luminosity in solar units from the absolute magnitude, and the projected separation simply from the coordinates. We have transformed the apparent magnitudes into solar luminosities by using $L_{\odot}=10^{0.4\left(m_{\odot}-m+D M\right)}$, where $m_{\odot}=4.83 \mathrm{mag}$ is the solar apparent magnitude in the $V$ band, $m$ is the apparent magnitude of the UDG, and $D M$ is the distance modulus to the host.

We also needed the rotation curve of the host galaxies to estimate their gravitational influence. In our sample, galaxies with an asymptotic circular velocity measurement are: NGC $1052\left(v_{\mathrm{rot}}=\right.$ $210 \mathrm{~km} \mathrm{~s}^{-1}$, van Gorkom et al. 1986), M $96\left(v_{\mathrm{rot}}=240 \mathrm{~km} \mathrm{~s}^{-1}\right.$, Moiseev et al. 2004), NGC $3628\left(v_{\text {rot }}=213 \mathrm{~km} \mathrm{~s}^{-1}\right.$, Wilding et al. 1993) NGC $1084\left(v_{\text {rot }}=140 \mathrm{~km} \mathrm{~s}^{-1}\right.$, Burbidge et al. 1963), NGC $2683\left(v_{\text {rot }}=215 \mathrm{~km} \mathrm{~s}^{-1}\right.$, Casertano \& van Gorkom 1991), NGC 4594/M $104\left(v_{\text {rot }}=350 \mathrm{~km} \mathrm{~s}^{-1}\right.$, Faber et al. 1977), NGC $7814\left(v_{\text {rot }}=215 \mathrm{~km} \mathrm{~s}^{-1}\right.$, Fraternali et al. 2011). From there, the external field at the position of the UDG can be estimated directly from the centripetal acceleration.

For elliptical galaxies where no rotation curve data is available, we converted the $K$ band luminosity using a Mass-to-Light $(M / L)$ ratio of 0.8 (de Blok et al. 2008) to get the total stellar mass. From there, the gravitational field at the distance of the UDG can be estimated in the context of MOND. This was the case for Cen A $(K=-23.9 \mathrm{mag}), \operatorname{NGC} 3625(K=-21.9 \mathrm{mag})$, NGC $3669(K=-22.3 \mathrm{mag}), \operatorname{NGC} 7814(K=-24.0 \mathrm{mag})$, NGC $5485(K=-23.8 \mathrm{mag})$, NGC $3384(K=-23.4 \mathrm{mag})$, M $105(K=-23.9 \mathrm{mag})$, NGC $5475(K=-22.8 \mathrm{mag})$, and NGC 3619 ( $K=-24.0$ mag) using the photometry by the Two Micron All Sky Survey (2MASS; Skrutskie et al. 2006) and a distance modulus given in Table 1.

Finally, for one face-on spiral galaxy (NGC 1042), no rotation curve was available. Therefore we first converted again its $K$ band luminosity using a $M / L$ ratio of 0.8 to get the stellar mass of the system. Then, we estimated the additional total gas mass with Eq. (2) from Di Cintio \& Lelli (2016) and added this to the stellar mass to get the total baryonic mass, and estimate the MOND external field at the position of the UDG from there.
Because galaxy group environments can host several large galaxies $\left(M_{V}<-19\right.$ mag $)$ we have to be careful to select the influencing galaxy when calculating the MONDian velocity dispersion of the UDG. If there is a bright galaxy apart from the dominant galaxy in the group - which is mostly indicated by the group's name - close to the UDG we have to reevaluate the MONDian velocity dispersion for those systems. A precise evaluation of the EFE as a function of the 3D position of the UDG in the group should then be performed using a numerical Poisson solver. In the present paper, we present an order of magnitude of the effect on the velocity dispersion by considering the EFE associated to each bright galaxy separately.

\subsection{Individual galaxy groups}

Below, we look more closely at the different galaxy groups and evaluate the impact of their different bright galaxies onto the UDGs.

NGC 1052 group: This galaxy group consists of three bright galaxies, the dominant elliptical galaxy NGC 1052, the faceon spiral galaxy NGC 1042, and the edge-on spiral galaxy NGC 1035. While NGC 1052-DF2 is only affected by the dominant elliptical, we note that NGC 1052-DF1 is at a separation of only $28 \mathrm{kpc}$ from NGC 1042, which indeed has a major impact on the predicted velocity dispersion. On the other hand, NGC 1052-DF4 is separated by only $21 \mathrm{kpc}$ from NGC 1035.

NGC 1084 group: To the north-east of the NGC 1052 group, a single spiral galaxy - NGC 1084 - resides well in isolation, bringing no additional complications to our calculations for NGC 1084-DF1.

M 96/Leo-I group: This group consists of seven bright galaxies (see e.g., Fig. 1 in Müller et al. 2018) at a mean distance of $10.7 \mathrm{Mpc}$. Of those, the two elliptical galaxies M 105 and NGC 3384 have separations of $53 \mathrm{kpc}$ and $67 \mathrm{kpc}$ from M96DF6, respectively. The large spiral galaxy M96 has a projected separation of $172 \mathrm{kpc}$. This UDG sits almost at the center of this massive group, complicating accurate predictions. Fortunately, for M 96-DF6, HST distance measurements are available $(D=10.2 \pm 0.3 \mathrm{Mpc}$; Cohen et al. 2018), indicating that this UDG is at the nominal distance of M96 $(D=10.4 \pm 0.3 \mathrm{Mpc}$; Karachentsev et al. 2013), and farther separated from M 105 ( $D=11.3 \pm 0.1 \mathrm{Mpc}$; Karachentsev et al. 2013) and NGC 3384 ( $D=9.4 \pm 0.1 \mathrm{Mpc}$; Karachentsev et al. 2013). Farther outside of this galaxy assembly resides dw1055+11.

Leo-Triplet: The Leo-Triplet is sometimes considered to be part of the Leo-I group, based on their same velocity and distance measurements. However, they are well separated on-sky into two distinct associations (Müller et al. 2018). The dominant edge-on spiral galaxy NGC 3628 is also the closest galaxy to the UDG dw1117+15, which is almost two degrees to the north of the system.

Cen A group: Cen A is the closest elliptical galaxy in the nearby universe and is the dominant galaxy in the Cen A group (Müller et al. 2017a). Because the distances to Cen A and Cen AMM-dw1 are well established with high-precision tip of the red giant branch measurements (Rejkuba 2004; Crnojević et al. 2019), no confusion is expected for their association.

NGC 5485 group: Several surveys have targeted the nearby M 101 group of galaxies and announced the discovery of new dwarf galaxy members (Merritt et al. 2014; Müller et al. 2017b; Bennet et al. 2017). Within its projected virial radius, reside 
Table 1. List of nearby ultra-diffuse galaxies within galaxy groups.

\begin{tabular}{|c|c|c|c|c|c|c|c|c|c|}
\hline Name & $\begin{array}{l}\text { RA } \\
\text { J2000 }\end{array}$ & $\begin{array}{l}\text { Dec } \\
\text { J2000 }\end{array}$ & $\begin{array}{l}m_{V} \\
\text { mag }\end{array}$ & $\begin{array}{l}\mu_{\mathrm{eff}, V} \\
\mathrm{mag} \operatorname{arcsec}^{-2}\end{array}$ & $\begin{array}{l}r_{\text {eff }} \\
\mathrm{kpc}\end{array}$ & $\begin{array}{l}\Delta_{\text {proj }} \\
\mathrm{kpc}\end{array}$ & $\begin{array}{l}(m-M) \\
\text { mag }\end{array}$ & $\begin{array}{l}L_{\odot} \\
10^{6} \\
\end{array}$ & Ref. \\
\hline NGC 7814-DGSAT-2 & 00:03:06.9 & $+16: 18: 30.8$ & 17.9 & 26.9 & 2.29 & 47 & 31.02 & 30.1 & Henkel et al. (2017) \\
\hline NGC 7814-DGSAT-7 & 00:00:44.0 & $+15: 27: 14.3$ & 18.7 & 27.0 & 1.49 & 258 & 31.02 & 14.4 & Henkel et al. (2017) \\
\hline NGC 1052-DF4 & 02:39:15.1 & $-08: 06: 58.6$ & 16.5 & 25.1 & 1.60 & 165 & 31.51 & 171.8 & Cohen et al. (2018) \\
\hline NGC 1052-DF1 & 02:40:04.6 & $-08: 26: 44.4$ & 18.2 & 27.4 & 2.51 & 109 & 31.51 & 35.9 & Cohen et al. (2018) \\
\hline NGC 1052-DF2 & $02: 41: 46.8$ & $-08: 24: 09.3$ & 16.2 & 25.1 & 2.06 & 79 & 31.51 & 226.5 & Cohen et al. (2018) \\
\hline NGC 1084-DF1 & $02: 42: 38.0$ & $-07: 20: 16.3$ & 16.2 & 24.7 & 1.63 & 280 & 31.34 & 193.8 & Cohen et al. (2018) \\
\hline NGC 2683-DGSAT-1 & $08: 52: 47.8$ & $+33: 47: 33.1$ & 14.7 & 26.5 & 4.10 & 68 & 30.05 & 234.5 & Javanmardi et al. (2016) \\
\hline NGC 2683-DGSAT-2 & $08: 55: 23.3$ & $+33: 33: 32.4$ & 16.3 & 25.8 & 1.39 & 105 & 30.05 & 53.7 & Javanmardi et al. (2016) \\
\hline M96-DF6 & $10: 46: 53.1$ & $+12: 44: 33.5$ & 16.6 & 27.0 & 2.11 & 172 & 30.15 & 44.9 & Cohen et al. (2018) \\
\hline $\mathrm{dw} 1055+11$ & $10: 55: 43.5$ & $+11: 58: 05.0$ & 16.9 & 26.7 & 1.78 & 411 & 30.15 & 34.0 & Müller et al. (2018) \\
\hline dw1117+15 & $11: 17: 02.1$ & $+15: 10: 17.0$ & 17.4 & 27.4 & 2.04 & 398 & 30.15 & 21.5 & Müller et al. (2018) \\
\hline NGC 3625-DGSAT-2 & $11: 21: 22.9$ & $+57: 34: 50.1$ & 20.3 & 26.9 & 1.54 & 151 & 32.86 & 18.0 & Henkel et al. (2017) \\
\hline NGC 3625-DGSAT-4 & $11: 21: 40.8$ & +572437.0 & 18.5 & 26.3 & 2.17 & 263 & 32.86 & 94.6 & Henkel et al. (2017) \\
\hline NGC 3625-DGSAT-3 & $11: 22: 12.0$ & $+58: 02: 11.9$ & 19.0 & 25.5 & 1.48 & 221 & 32.86 & 59.7 & Henkel et al. (2017) \\
\hline NGC 3669-DGSAT-2 & $11: 24: 48.3$ & $+57: 37: 58.0$ & 20.0 & 26.2 & 1.86 & 93 & 33.17 & 31.4 & Henkel et al. (2017) \\
\hline NGC 3669-DGSAT-3 & $11: 26: 38.8$ & $+57: 41: 19.1$ & 18.3 & 26.9 & 3.91 & 123 & & 150.4 & Henkel et al. (2017) \\
\hline NGC 4594-DGSAT-1 & $12: 39: 55.1$ & $-11: 44: 38.4$ & 16.2 & 26.1 & 1.67 & 24 & 30.12 & 62.8 & Javanmardi et al. (2016) \\
\hline CenA-MM-Dw1 & $13: 30: 14.3$ & $-41: 53: 34.8$ & 14.2 & 25.4 & 1.82 & 92 & 27.84 & 48.9 & Crnojević et al. (2019) \\
\hline M101-DF5 & $14: 04: 28.1$ & $+55: 37: 00.0$ & 17.7 & 27.7 & 4.90 & 342 & 32.16 & 103.0 & Merritt et al. (2016) \\
\hline M101-DF7 & $14: 05: 47.5$ & $+55: 07: 57.3$ & 20.1 & 28.4 & 2.60 & 112 & 32.16 & 11.3 & Merritt et al. (2016) \\
\hline M101-DF4 & $14: 07: 33.8$ & $+54: 42: 39.2$ & 18.5 & 27.6 & 3.60 & 140 & 32.16 & 49.3 & Merritt et al. (2016) \\
\hline M101-DF6 & $14: 08: 18.7$ & $+55: 11: 30.6$ & 19.8 & 27.5 & 2.90 & 117 & 32.16 & 14.9 & Merritt et al. (2016) \\
\hline
\end{tabular}

the two massive elliptical galaxies - NGC 5485 and NGC 5473 with similar mass estimates - at a distance of $27 \mathrm{Mpc}$. Some of the putative M 101 members have recently been identified as background UDG members of the NGC 5485 group by their non-detected red giant branch star population in HST observations (Merritt et al. 2016). Many more candidates await distance measurements and can potentially be UDG members of the NGC 5485 group as well. While M 101-DF4, M 101-DF6, and M 101-DF7 are in projection closer to NGC 5485 such that it is reasonable to use this galaxy as the major external field provider, the case of M 101-DF5 is less clear. It has an on-sky separation of 44 arcmin to NGC 5485, and only 10 arcmin and 23 arcmin to the other nearby galaxies NGC 5475 and NGC 5443, respectively. These two galaxies have the same systemic velocity measurement as the NGC 5485 group, which could indicate that M 101DF5 could be associated to these these two galaxies, and not to NGC 5485 itself. We therefore consider two cases for M 101DF5, (a) one case where it resides within NGC 5485 external field, and (b) another where it resides within NGC 5475 external field.

NGC 2683 group: This galaxy group is made up of one large spiral galaxy and resides in isolation, making the predictions for NGC 2683-DGSAT-1 and NGC 2683-DGSAT-2 straightforward.

NGC 4594/M 104 group: This famous galaxy group with the M 104 (the Sombrero galaxy) in its heart is again, very isolated, making the prediction for NGC 4594-DGSAT-1 straightforward.

NGC 3625 group: The isolated spiral galaxy NGC 3625 is in projection very close to the shell galaxy NGC 3619, and the elliptical galaxy NGC 3613 which are well separated in velocity space from NGC 3625. It is therefore difficult to disentangle the membership of the UDGs between those groups. If NGC 3625-DGSAT-2 is a NGC 3625 member, the external field of NGC 3625 is almost negligible, however, if it is associated to NGC $3619(D=25 \pm 1.8 \mathrm{Mpc})$, it is deep in the quasi-Newtonian regime. For the other two UDGs in this region (NGC 3625DGSAT-3, NGC 3625-DGSAT-4), the separation to NGC 3619 is too large to be strongly affect by the EFE.

NGC 3669 group: The isolated spiral galaxy NGC 3669 is to the east of the NGC 3625 and NGC 3619 groups. However, their separation is too large for major confusions, allowing straightforward predictions for NGC 3669-DGSAT-2 and NGC 3669DGSAT-3.

NGC 7184 group: This group again is well isolated, with the spiral galaxy NGC 7184 at its center, making the predictions for NGC 7814-DGSAT-2 and NGC 7814-DGSAT-7 straightforward.

\section{Predicted velocity dispersions}

For all galaxies of Table 1 we have first calculated whether the external field dominates the dynamics or not. To make a distinction between the isolated and the quasi-Newtonian regimes we estimate the fraction $g / g_{\text {ex }}$ as a function of the three dimensional separation of the UDG to its main host (or to various possible hosts), up to one Megaparsec. We can safely assume that, when the internal acceleration is less than twice the external one, the EFE will play a role. In such a case, we used the $\sigma$ as previously derived by including the EFE in Eqs. (4)-(7). Above this value of the internal to external field ratio, the galaxy can be assumed to be isolated, therefore $\sigma_{\text {iso }}$ applies as in Eq. (3).

In Fig. 1 we show the predictions for the 18 UDGs in our sample which have one clear host. The curves start at the projected separation of the UDG to its host. We derived upper and lower limits for the curves as follows: for every UDG, we vary the $V$-band $M / L$ ratio between 1 and 4 , as well as take into account the distance uncertainty to the host. We also allow the mass of the host to vary by $\pm 20 \%$. The lower limit is therefore given by a $V$ band $M / L=1$, a distance to the host at its lowest limit (making the UDG less luminous), and the $+20 \%$ 
O. Müller et al.: Predicted MOND velocity dispersions for a catalog of ultra-diffuse galaxies in group environments
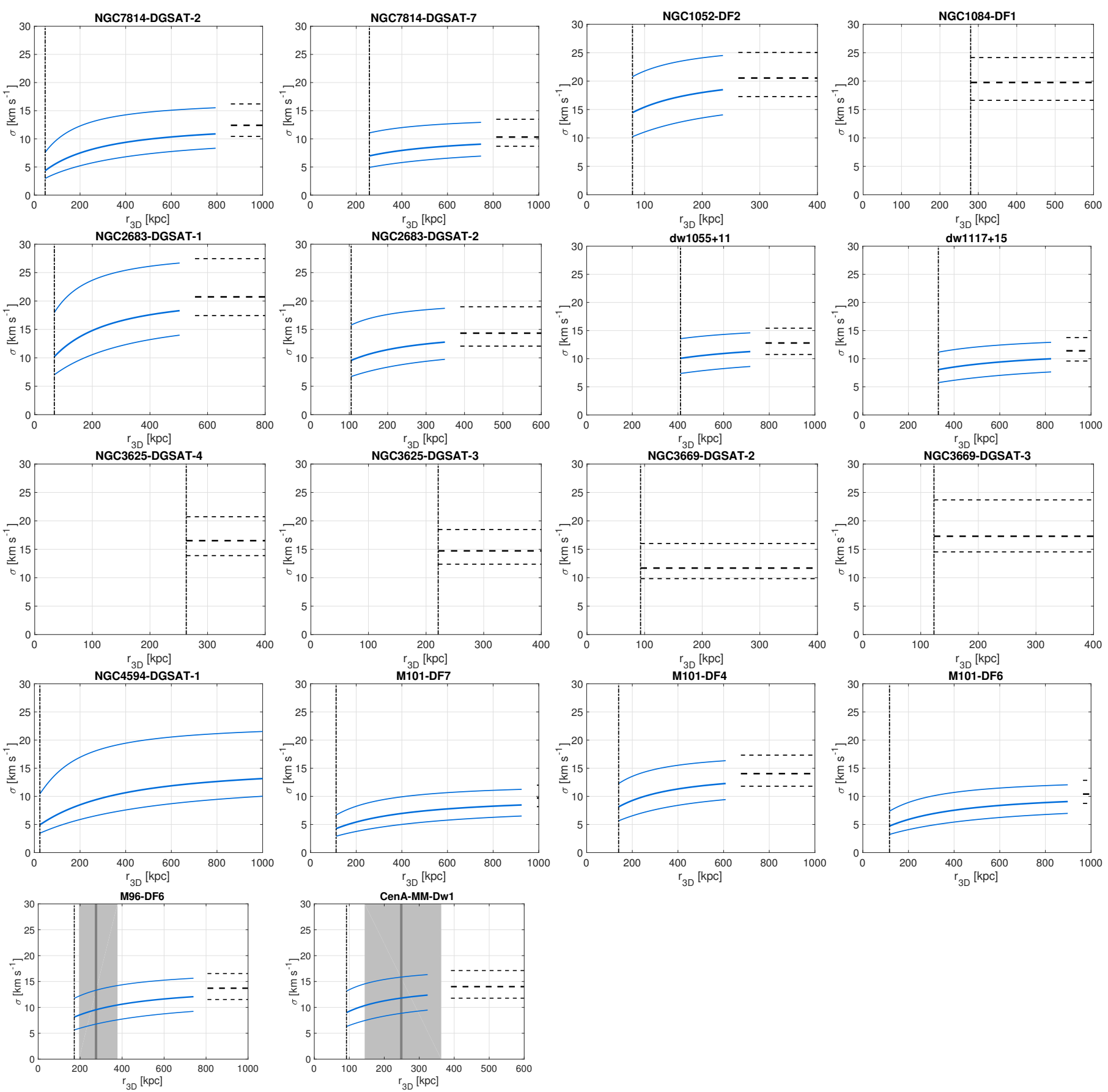

Fig. 1. Predicted velocity dispersions including the EFE (blue lines) and for the isolated case (dotted lines) as function of the three dimensional separation up to $1 \mathrm{Mpc}$. The inclusion of the EFE is stopped when $g / g_{\mathrm{ex}}>2$. The vertical dotted line corresponds to the on-sky separation (and thereby lower limit of the 3D distance) between the host and the UDG. The upper and lower limits of the curves are derived by varying the $M / L$ and the distance to the host, see text. The last two entries - M96-DF6 and Cen A-MM-Dw1 - have high-precision distance measurements available, we therefore indicate the three dimensional separation (gray line) as well as the associated $1 \sigma$ uncertainties (gray area).

upper limit for the host mass, whilst the upper limit is given by a $V$ band $M / L=4$, the upper limit for the host galaxy system distance (making the UDG more luminous), and the $-20 \%$ lower limit for the host mass. We also give a typical estimate in between those two curves, with $M / L=2$ and the literature values for the distance and mass (or rotational velocity) of the host. We have simplified the Figures by using the transition $g / g_{\text {ex }}=2$ for the typical case also for the transition for the lower and upper limits. Correctly we would need to take into account that in the lower case, the EFE will have a larger range until the
UDG reaches the regime of isolation. We also left a gap between the $\sigma$ estimation including the EFE and the isolated case. This is caused by the fact that we would need to carefully interpolate between the two regimes, which is not the focus of this work.

For four UDGs in our catalog, the host dominating the EFE is ambiguous. For NGC 3625-DGSAT-2 the two possible host galaxies are not even within the same group and have therefore different nominal distances. For the others, to make a precise prediction, one would need to use a numerical Poisson solver as a function of the full 3D configuration of the galaxy group. 

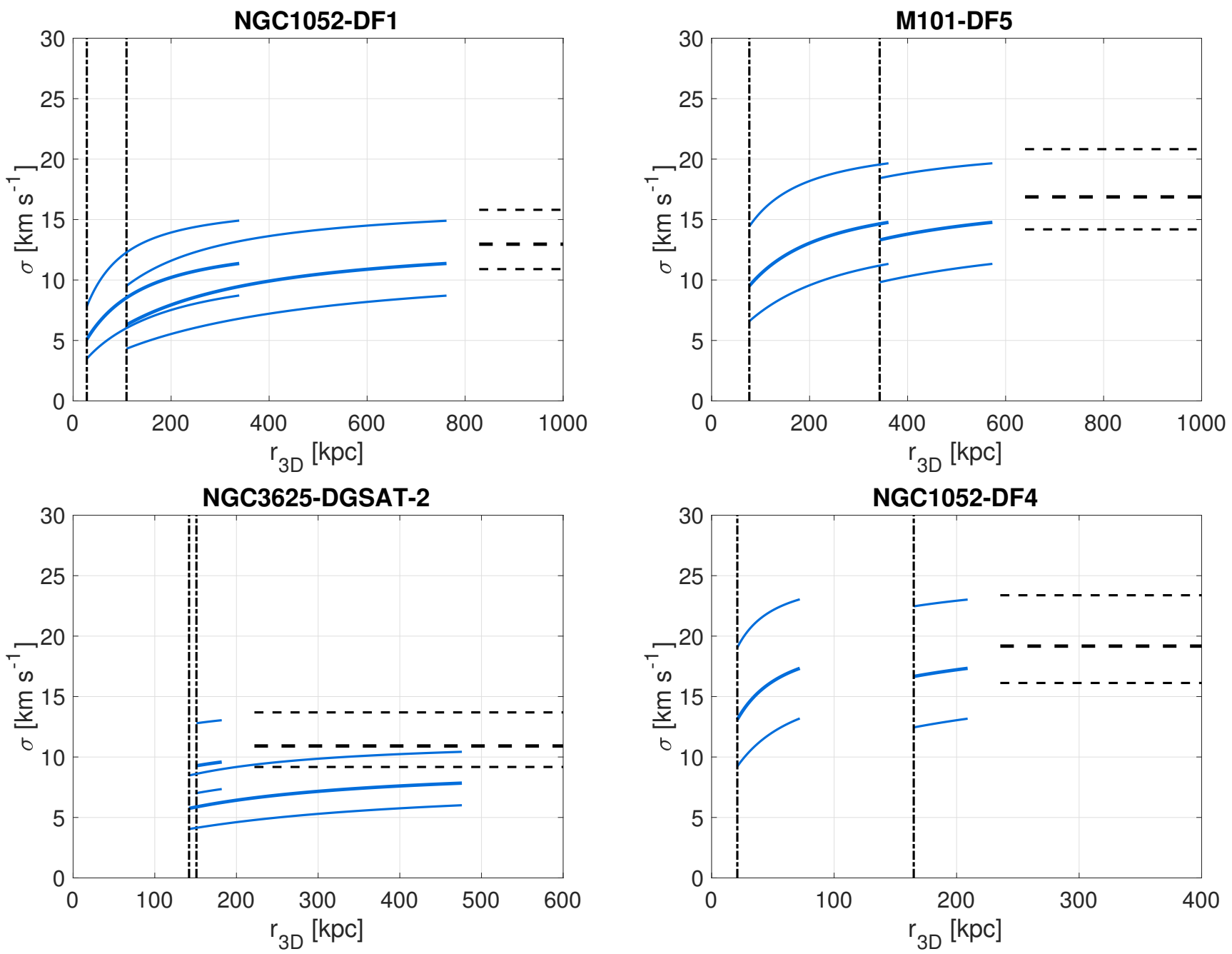

Fig. 2. Continuation of Fig. 1, in the cases where multiple external fields have a potentially strong influence on the ultra-diffuse galaxies. For NGC 3625-DGSAT-2 the two host galaxies are not within the same group and have therefore different nominal distances.

To give an idea of the range of possible predictions, we provide in Fig. 2 the predictions as a function of the separation to each potential host galaxy separately.

In Table 2 we present the minimal and maximal allowed values for the velocity dispersion, as well as a typical value for the UDG, if it has a $V$ band $M / L$ ratio of two (which is typical for old dwarf galaxies) and has a physical distance of $\sqrt{3 / 2} \cdot \Delta_{\text {proj. }}$. This distance simply assumes that the depth along the line-of-sight is the same as the on-sky separation. For 17 of our 22 UDGs, we note that the EFE was indeed important to consider and will effectively lower the stellar velocity dispersion of the galaxy for most of its possible 3D separations.

\section{Discussion}

The discovery of the UDG NGC 1052-DF2 with its spectroscopic follow-up observations opened up a novel opportunity to study MOND and the EFE in this class of galaxies. In Fig. 3 we present the MONDian calculation for this galaxy, this time including the $\sigma$ measurements by van Dokkum et al. (2018) and Emsellem et al. (2018). The former gave a velocity dispersion of $\sigma=3.2_{-3.2}^{+5.5} \mathrm{~km} \mathrm{~s}^{-1}$ (shown in red in Fig. 3), using globular clusters as tracer of the stellar body of the system. It is apparent that such a low value for the velocity dispersion would indeed be an outlier in MOND (to be more precise, a $2 \sigma$ outlier, see Fig. 1 in Kroupa et al. 2018). Emsellem et al. (2018), using precise IFU spectroscopy - obtained with MUSE mounted at the Very Large Telescope - directly derived a stellar velocity dispersion of $\sigma=16.3_{-5.0}^{+5.0} \mathrm{~km} \mathrm{~s}^{-1}$ (shown in gray in Fig. 3). This value is in excellent agreement with the MONDian estimates made here and in previous studies (Kroupa et al. 2018; Famaey et al. 2018). Additionally, Emsellem et al. (2018) found two more globular clusters and three planetary nebulae. Using them, and updated velocities for five previously known globular clusters, they derive a velocity dispersion of $\sigma=10.5_{-2.2}^{+4.0} \mathrm{~km} \mathrm{~s}^{-1}$, which is at the lower limit of allowed values in MOND (see Table 4).

Assuming that the stellar velocity dispersion $\sigma=$ $16.3 \mathrm{~km} \mathrm{~s}^{-1}$ is the true value for this system, and that we live in a MONDian universe, NGC 1052-DF2 is then most likely at a physical separation of $124 \mathrm{kpc}$ to its host NGC 1052, considering the external field. This does make insofar sense, that this is a typical radial separation of a satellite to its host. However, NGC 1052-DF2's systemic velocity is off by $300 \mathrm{~km} \mathrm{~s}^{-1}$, which could indicate that this galaxy is not a bound member of the NGC 1052 group. Only precise distance measurements can tell whether this galaxy indeed belongs to the group, or whether it is a field galaxy in the fore-or background. In the latter case, an estimate with the corresponding distance would be needed to check the validity of MOND. 
Table 2. Predicted velocity dispersions.

\begin{tabular}{lcll}
\hline \hline Name & $\begin{array}{c}\sigma_{\text {typical }} \\
\mathrm{km} \mathrm{s}^{-1}\end{array}$ & $\begin{array}{l}\sigma_{\text {min }} \\
\mathrm{km} \mathrm{s}^{-1}\end{array}$ & $\begin{array}{l}\sigma_{\text {max }} \\
\mathrm{km} \mathrm{s}^{-1}\end{array}$ \\
\hline NGC 7814-DGSAT-2 & 4.6 & 3.0 & 16.2 \\
NGC 7814-DGSAT-7 & 7.4 & 4.9 & 13.5 \\
NGC 1052-DF4 & 17.3 & $9.2^{a}$ & 23.4 \\
NGC 1052-DF1 & 6.8 & $3.7^{a}$ & 15.8 \\
NGC 1052-DF2 & 15.3 & 10.2 & 25.1 \\
NGC 1084-DF1 & 19.8 & 16.6 & 24.1 \\
NGC 2683-DGSAT-1 & 10.9 & 7.0 & 27.4 \\
NGC 2683-DGSAT-2 & 10.1 & 6.7 & 19.0 \\
M 96-DF6 & 8.7 & 5.6 & 16.5 \\
dw1055+11 & 10.5 & 7.4 & 15.4 \\
dw1117+15 & 8.5 & 5.7 & 13.7 \\
NGC 3625-DGSAT-2 & 9.6 & 7.0 & 13.7 \\
& 6.1 & 4.0 & 11.0 \\
NGC 3625-DGSAT-4 & 16.5 & 13.9 & 20.7 \\
NGC 3625-DGSAT-3 & 14.7 & 12.4 & 18.5 \\
NGC 3669-DGSAT-2 & 11.7 & 9.8 & 16.0 \\
NGC 3669-DGSAT-3 & 17.3 & 14.5 & 23.7 \\
NGC 4594-DGSAT-1 & 5.1 & 3.5 & 21.5 \\
Cen A-MM-Dw1 & 9.6 & 6.3 & 17.1 \\
M 101-DF5 & 13.9 & $6.6^{a}$ & 20.8 \\
M 101-DF6 & 5.1 & 3.2 & 12.8 \\
M 101-DF4 & 8.4 & 5.6 & 17.3 \\
M 101-DF7 & 4.6 & 2.9 & 12.0 \\
\hline
\end{tabular}

Notes. ${ }^{(a)}$ Denotes that potentially the ultra-diffuse galaxy is embedded in multiple strong external fields, in which case we present the smallest velocity dispersion value from all of them. ${ }^{(b)}$ The values here are slightly higher than in Famaey et al. (2018) because of very slightly different assumptions for the baryonic mass of the UDG and host, as well as in the definition of the upper limit. ${ }^{(c)}$ NGC 3625-DGSAT-2 could also be associated to NGC 3619 (second shown entry) instead of NGC 3625 (first shown entry), which is closer to us, therefore we provide the predictions for both cases.

The MUSE observations are indeed an encouraging prospect for future studies of these UDGs. At these low-surface brightness levels, it is possible to get an estimate of the velocity dispersion under ten hours of observation time, which is expensive but certainly doable. Other today's available facilities which potentially can conduct such studies are KCWI installed at Keck and Megara at the Gran Canaria Telescope. In the future, the next-generation telescopes like the ELT will certainly be able to conduct such measurements in short times for a large sample of galaxies.

We refrain here from making any predictions of the velocity dispersion of the UDGs in terms of the standard $\triangle \mathrm{CDM}$ framework. In principle, we could collect the dark matter halos in high-resolution simulations for the satellite galaxies, given the observed luminosity and construct a range of possible values for the velocity dispersion. However, the origin of UDGs is still not understood. If they are failed Milky Way type galaxies, they will possess a large dark matter halo to begin with, and on the other hand, if they origin from dwarf galaxies, they will reside in smaller dark matter halos. This huge range of possibilities makes it unfeasible to make a prediction of the velocity dispersion in the standard context.

We note that two of the UDGs within our sample Cen A-MM-Dw1 and M 96-DF6 - already have precise distance measurements available (Crnojević et al. 2019; Cohen et al. 2018). The three dimensional separation between Cen $A$ and

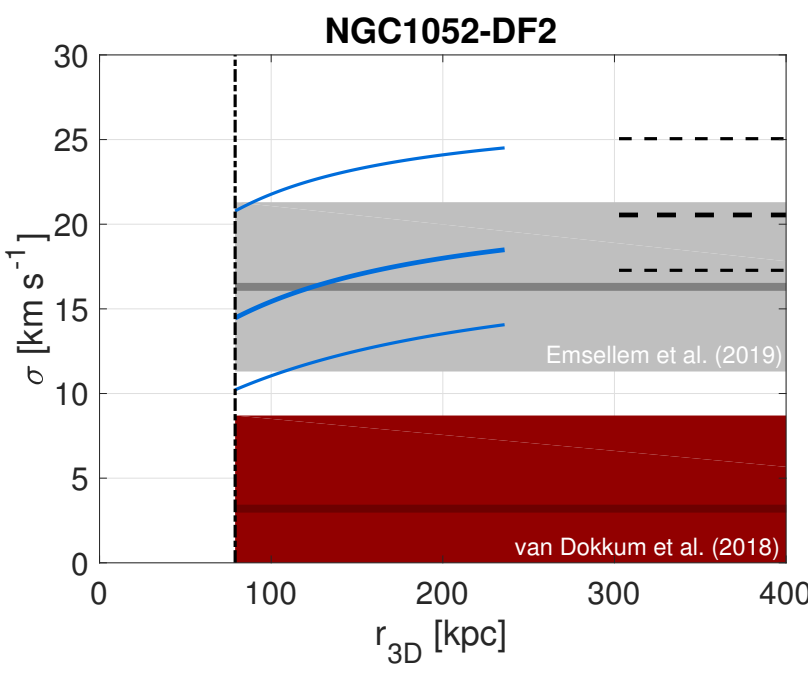

Fig. 3. Velocity dispersions for the ultra-diffuse galaxy NGC 1052-DF2. The blue and black lines correspond to the MOND predictions as in Fig. 1 . The red area denotes the $1 \sigma$ range of the velocity dispersion measurement of van Dokkum et al. (2018), the gray are the stellar velocity dispersion measurement of Emsellem et al. (2018).

Cen A-MM-dw1 is $249 \mathrm{kpc}$ with a distance uncertainty for Cen A-MM-dw1 of $\pm 120 \mathrm{kpc}$. This galaxy resides at a separation where the EFE plays a role. In Fig. 1 we indicate the measured as well as the allowed $1 \sigma$ separations derived from the errors. Assuming that Cen-A-MM-Dw1 follows our typical estimates, it should have a velocity dispersion of $\sigma=$ $11.8_{-15}^{+2.2} \mathrm{~km} \mathrm{~s}^{-1}$. The upper limit is given by the isolated case. For M96-DF6, the three dimensional separation between M96 and M 96-DF6 is $276 \mathrm{kpc}$, with a distance uncertainty for M96DF6 of $\pm 300 \mathrm{kpc}$. This gives a typical MONDian velocity dispersion of $\sigma=9.5_{-1.0}^{+1.0} \mathrm{~km} \mathrm{~s}^{-1}$. For these estimates, we have not varied the distance to the host. Once taken into account, this will additionally increase the allowed interval for the velocity dispersion.

With the updated velocity dispersion for NGC 1052-DF2 at hand, within the $\Lambda C D M$ paradigm it is possible to estimate the total mass (dark matter + baryonic mass) with Eq. (2) given in Wolf et al. (2010). With a stellar velocity dispersion of $\sigma=$ $16.3 \mathrm{~km} \mathrm{~s}^{-1}$ we get a mass within $r_{1 / 2}$ of $5.08 \times 10^{8} M_{\odot}$. With an absolute magnitude of $-15.3 \mathrm{mag}$ in the $V$ band the galaxy has a total baryonic mass of $2.27 \times 10^{8} M_{\odot}$ (using a $M / L=2$ and a $V$ band magnitude of 4.83 for the Sun), leading to a dynamical to visible mass ratio of 4.5 within the half-light radius. This rough estimate is well in agreement with Local Group dwarfs in the corresponding luminosity regime (see McConnachie 2012). Therefore, the observed velocity dispersion of NGC 1052-DF2 is not peculiar, and hence comfortable in both paradigms, the $\Lambda \mathrm{CDM}$ standard model of cosmology and the alternative gravity paradigm (MOND).

Although NGC 1052-DF2 cannot help us to distinguish between MOND and $\Lambda C D M$, there may be other galaxies in our sample which do. The most affected galaxy in terms of the EFE in our sample $-\max \left(\sigma_{\text {iso }} / \sigma_{\text {typical }}\right)-$ is NGC $4594-D G S A T-1$. Let us make a thought experiment, in which we live in a MONDian universe, but fail to realize the fact, and thus describe it in terms of Newtonian dynamics. Assume that the calculated typical velocity dispersion correctly describes the UDGs, and thus that NGC 4594-DGSAT-1 has a typical velocity dispersion of $\sigma=5.8 \mathrm{~km} \mathrm{~s}^{-1}$. Now our Newtonian observers have exactly 
measured this value to infinite precision, and using it with Wolf et al. (2010) they will derive a mass within $r_{1 / 2}$ of $5.0 \times$ $10^{7} M_{\odot}$. The mass of the baryonic content of the galaxy inferred from the light is $6 \times 10^{7} M_{\odot}$, giving a dynamical to visible mass ratio within $r_{1 / 2}$ of only 1.7, and hence a dynamical mass-to-light ratio $M_{d y n} / L$ of 3.4. For a galaxy with $M_{V}=-13.9$ mag this is similar to Milky Way dwarf satellite Leo-I (Mateo et al. 2008), sitting at the lower end of the luminosity- $M_{\text {dyn }} / L$ relation (see Fig. 11 in McConnachie 2012). However, if the lowest possible value in MOND was measured, i.e., $\sigma_{\min }=3.5 \mathrm{~km} \mathrm{~s}^{-1}$, the Newtonian observers would derive a $M_{\mathrm{dyn}} / L$ ratio of only 1.2 , clearly too small for primordial dwarf galaxies in $\Lambda \mathrm{CDM}$. This simple thought experiment shows that we can, in principle, distinguish between primordial dwarf galaxies in the $\Lambda \mathrm{CDM}$ framework and the MOND predictions for the cases where the UDG is strongly affected by the EFE and high-precision distance and velocity dispersions measurements are available. Let us note that such a situation has already happened for some Andromeda dwarfs (McGaugh \& Milgrom 2013a,b) and Crater-II (McGaugh 2016). As these previous examples show, such an observation would of course not falsify $\Lambda \mathrm{CDM}$, but would pose interesting challenges to it. In terms of falsifying MOND with such objects, as discussed in Famaey et al. (2018), an issue could always be whether the internal dynamics of a UDG on an eccentric orbit have time to come into equilibrium with the continually changing external field. Hence, once an interesting object challenging MOND is found, elucidating this issue would necessitate more involved simulations.

On the other hand, to tentatively falsify MOND, it would be worthwhile to follow up some of the UDGs for which we have shown that the EFE plays a minor role, or no role at all. In that case, when their velocity dispersion is measured to be lower than the MOND prediction for the isolated case, then there is not much room in MOND for those velocity dispersions to vary (assuming that the values used for the predictions like the distance and luminosity are correct). This is in contrast to the ultra-faint dwarf galaxies in the Milky Way, which MOND tidal effects would certainly bring out of equilibrium, complicating any comparison between observations and predictions (McGaugh \& Wolf 2010; Fattahi et al. 2018; Read et al. 2019).

\section{Summary and conclusion}

Ultra-diffuse galaxies are puzzling objects for cosmology. While they resemble galaxies like the Milky Way in size, they are as faint and feeble as dwarf galaxies. In MOND - when they are close to a large galaxy - they can be affected by its external field effect, making them quasi-Newtonian. This EFE - unique to MOND - will lower the velocity dispersion of these systems, when compared to isolation. We have compiled a catalog of UDGs in group environments in the nearby universe to predict their velocity dispersions. We used an analytic expression to include the external field in our calculations and derived velocity dispersions as function of the true three dimensional separation of the UDGs from their host. When the internal accelerations of the UDGs overrule the external effects, we present the prediction based on simple MOND formula for isolated objects. By varying the mass to light ratio of the UDG, as well as the distance to its putative host, we give upper and lower limits allowed from MOND. Future observations of these UDGs can therefore test the validity of MOND and test the external field effect.

Two of our UDGs - Cen A-MM-Dw1 and M 96-DF6 - have high-precision distance measurements with the HST. In these cases, we have calculated the three dimensional distances to their host and made a more narrow prediction of the expected velocity dispersion in MOND, assuming that they follow a typical $M / L$ ratio of two. For Cen A-MM-Dw1 we expect a velocity dispersion of $\sigma=11.8_{-1.5}^{+2.2} \mathrm{~km} \mathrm{~s}^{-1}$, and for M 96-DF6 a velocity dispersion of $\sigma=9.5_{-1.0}^{+1.0} \mathrm{~km} \mathrm{~s}^{-1}$.

For NGC 1052-DF2 high-precision IFU data have become available, taken with VLT+MUSE. This is the first time the stellar velocity dispersion itself of an UDG has been measured. We compared this new measurement to the MOND prediction, already made in Famaey et al. (2018), Kroupa et al. (2018), and find excellent agreement. This is an encouraging prospect, as it shows that testing our predictions is feasible with modern instruments.

As a last caveat, we should mention that the predictions made here make rather simple assumptions, such as no strong anisotropy, no rotation on the sky, and an analytic MOND estimate with only one interpolating function and with an implicitly small eccentricity for the UDG orbit. As discussed in Famaey et al. (2018), a UDG on a very eccentric orbit with a continually changing external field could display a lower velocity dispersion than the prediction made here. Hence, once an interesting object challenging MOND is found, more involved simulations will be necessary to elucidate this issue.

Acknowledgements. O.M. thanks the Swiss National Science Foundation for financial support. We thank the referee for their useful and constructive report.

\section{References}

Amorisco, N. C., \& Loeb, A. 2016, MNRAS, 459, L51

Begeman, K. G., Broeils, A. H., \& Sanders, R. H. 1991, MNRAS, 249, 523 Bekenstein, J., \& Milgrom, M. 1984, ApJ, 286, 7

Bennet, P., Sand, D. J., Crnojević, D., et al. 2017, ApJ, 850, 109 Burbidge, E. M., Burbidge, G. R., \& Prendergast, K. H. 1963, ApJ, 137, 376 Caldwell, N., Walker, M. G., Mateo, M., et al. 2017, ApJ, 839, 20

Carlin, J. L., Sand, D. J., Price, P., et al. 2016, ApJ, 828, L5 Casertano, S., \& van Gorkom, J. H. 1991, AJ, 101, 1231

Chan, T. K., Kereš, D., Wetzel, A., et al. 2018, MNRAS, 478, 906 Cohen, Y., van Dokkum, P., Danieli, S., et al. 2018, ApJ, 868, 96 Cortés, R. A. M., \& Hernandez, X. 2017, MNRAS, 470, 1086 Crnojević, D., Sand, D. J., Caldwell, N., et al. 2014, ApJ, 795, L35 Crnojević, D., Sand, D. J., Bennet, P. N., et al. 2019, ApJ, 872, 80 de Blok, W. J. G., Walter, F., Brinks, E., et al. 2008, AJ, 136, 2648 Di Cintio, A., \& Lelli, F. 2016, MNRAS, 456, L127

Di Cintio, A., Brook, C. B., Dutton, A. A., et al. 2017, MNRAS, 466, L1 Eigenthaler, P., Puzia, T. H., Taylor, M. A., et al. 2018, ApJ, 855, 142

Emsellem, E., van der Burg, R. F. J., Fensch, J., et al. 2018, A\&A, submitted [arXiv:1812.07345]

Faber, S. M., Balick, B., Gallagher, J. S., \& Knapp, G. R. 1977, ApJ, 214, 383 Famaey, B., \& Binney, J. 2005, MNRAS, 363, 603

Famaey, B., \& McGaugh, S. S. 2012, Liv. Rev. Rel., 15, 10

Famaey, B., McGaugh, S., \& Milgrom, M. 2018, MNRAS, 480, 473

Fattahi, A., Navarro, J. F., Frenk, C. S., et al. 2018, MNRAS, 476, 3816

Fensch, J., van der Burg, R. F. J., Jerabkova, T., et al. 2018, A\&A, accepted [arXiv:1812.07346]

Fraternali, F., Sancisi, R., \& Kamphuis, P. 2011, A\&A, 531, A64 Gentile, G., Famaey, B., Combes, F., et al. 2007, A\&A, 472, L25

Gentile, G., Angus, G. W., Famaey, B., Oh, S.-H., \& de Blok, W. J. G. 2012, A\&A, 543, A47

Greco, J. P., Greene, J. E., Strauss, M. A., et al. 2018, ApJ, 857, 104

Haghi, H., Bazkiaei, A. E., Zonoozi, A. H., \& Kroupa, P. 2016, MNRAS, 458, 4172

Henkel, C., Javanmardi, B., Martínez-Delgado, D., Kroupa, P., \& Teuwen, K. 2017, A\&A, 603, A18

Impey, C., Bothun, G., \& Malin, D. 1988, ApJ, 330, 634

Javanmardi, B., Martinez-Delgado, D., Kroupa, P., et al. 2016, A\&A, 588, A89

Karachentsev, I. D., Makarov, D. I., \& Kaisina, E. I. 2013, AJ, 145, 101

Kroupa, P., Haghi, H., Javanmardi, B., et al. 2018, Nature, 561, E4

Laporte, C. F. P., Agnello, A., \& Navarro, J. F. 2019, MNRAS, 484, 245

Lelli, F., Duc, P.-A., Brinks, E., et al. 2015, A\&A, 584, A113 
O. Müller et al.: Predicted MOND velocity dispersions for a catalog of ultra-diffuse galaxies in group environments

Londrillo, P., \& Nipoti, C. 2009, Mem. Soc. Astron. It. Suppl., 13, 89 Martin, N. F., Collins, M. L. M., Longeard, N., \& Tollerud, E. 2018, ApJ, 859, L5

Mateo, M., Olszewski, E. W., \& Walker, M. G. 2008, ApJ, 675, 201

McConnachie, A. W. 2012, AJ, 144, 4

McGaugh, S. S. 2016, ApJ, 832, L8

McGaugh, S., \& Milgrom, M. 2013a, ApJ, 766, 22

McGaugh, S., \& Milgrom, M. 2013b, ApJ, 775, 139

McGaugh, S. S., \& Wolf, J. 2010, ApJ, 722, 248

Merritt, A., van Dokkum, P., \& Abraham, R. 2014, ApJ, 787, L37

Merritt, A., van Dokkum, P., Danieli, S., et al. 2016, ApJ, 833, 168

Mihos, J. C., Durrell, P. R., Ferrarese, L., et al. 2015, ApJ, 809, L2

Mihos, J. C., Harding, P., Feldmeier, J. J., et al. 2017, ApJ, 834, 16

Mihos, J. C., Carr, C. T., Watkins, A. E., Oosterloo, T., \& Harding, P. 2018, ApJ, 863, L7

Milgrom, M. 1983, ApJ, 270, 365

Milgrom, M. 2018, Phys. Rev. D, 98, 104036

Milgrom, M. 2019, Phys. Rev. D, 99, 044041

Moiseev, A. V., Valdés, J. R., \& Chavushyan, V. H. 2004, A\&A, 421, 433

Müller, O., Jerjen, H., \& Binggeli, B. 2015, A\&A, 583, A79

Müller, O., Jerjen, H., \& Binggeli, B. 2017a, A\&A, 597, A7
Müller, O., Scalera, R., Binggeli, B., \& Jerjen, H. 2017b, A\&A, 602, A119

Müller, O., Jerjen, H., \& Binggeli, B. 2018, A\&A, 615, A105

Pawlowski, M. S., \& McGaugh, S. S. 2014, MNRAS, 440, 908

Pawlowski, M. S., McGaugh, S. S., \& Jerjen, H. 2015, MNRAS, 453, 1047

Read, J. I., Walker, M. G., \& Steger, P. 2019, MNRAS, 484, 1401

Rejkuba, M. 2004, A\&A, 413, 903

Sandage, A., \& Binggeli, B. 1984, AJ, 89, 919

Skrutskie, M. F., Cutri, R. M., Stiening, R., et al. 2006, AJ, 131, 1163

Thomas, G. F., Famaey, B., Ibata, R., et al. 2018, A\&A, 609, A44

Toloba, E., Lim, S., Peng, E., et al. 2018, ApJ, 856, L31

Torrealba, G., Koposov, S. E., Belokurov, V., \& Irwin, M. 2016, MNRAS, 459, 2370

van der Burg, R. F. J., Muzzin, A., \& Hoekstra, H. 2016, A\&A, 590, A20

van Gorkom, J. H., Knapp, G. R., Raimond, E., Faber, S. M., \& Gallagher, J. S. 1986, AJ, 91, 791

van Dokkum, P. G., Abraham, R., Merritt, A., et al. 2015, ApJ, 798, L45

van Dokkum, P., Danieli, S., Cohen, Y., et al. 2018, Nature, 555, 629

Venhola, A., Peletier, R., Laurikainen, E., et al. 2017, A\&A, 608, A142

Wilding, T., Alexander, P., \& Green, D. A. 1993, MNRAS, 263, 1075

Wolf, J., Martinez, G. D., Bullock, J. S., et al. 2010, MNRAS, 406, 1220

Yozin, C., \& Bekki, K. 2015, MNRAS, 452, 937 